Jacek Hejduk, Institute of Mathematics, Łódź University, ul. Stefana

Banacha 22, 90-238 Łódź, Poland.

\title{
ON THE DENSITY TOPOLOGY WITH RESPECT TO AN EXTENSION OF LEBESGUE MEASURE
}

\begin{abstract}
We prove that, for every complete extension $\mu$ of Lebesgue measure, the $\mu$-density topology is the Hashimoto topology generated by the density topology and the $\sigma$-ideal of $\mu$-null sets (cf. [1]).
\end{abstract}

Let $\mu$ be any complete extension of Lebesgue measure $l$ on the real line $\mathbb{R}$. Let $\mathcal{S}_{\mu}$ denote the $\sigma$-field of $\mu$-measurable sets, $\mathcal{I}_{\mu}$ - the $\sigma$-ideal of $\mu$-null sets. We denote by $\mathcal{L}$ the $\sigma$-field of Lebesgue measurable sets. Let $\mu^{*}$ and $\mu_{*}$ be, respectively, the outer measure and the inner measure induced by $\mu$. We recall that a point $x \in \mathbb{R}$ is a $\mu$-density point of a $\mu$-measurable set $X$ if

$$
\lim _{h \rightarrow 0+} \frac{\mu(X \cap[x-h, x+h])}{2 h}=1 .
$$

For each set $X \in \mathcal{S}_{\mu}$, let

$$
\Phi_{\mu}(X)=\{x \in \mathbb{R}: x \text { is a } \mu \text {-density point of } X\}
$$

Let

$$
\mathcal{T}_{\mu}^{*}=\left\{X \in \mathcal{S}_{\mu}: X \subset \Phi_{\mu}(X)\right\}
$$

Theorem 1 (cf. [2]) The family $\mathcal{T}_{\mu}^{*}$ is a topology in $\mathbb{R}$.

Proof. It is clear that the sets $\emptyset$ and $\mathbb{R}$ are members of the family $\mathcal{T}_{\mu}^{*}$. Also, the family $\mathcal{T}_{\mu}^{*}$ is closed under finite intersections. Our task is to prove that, for each family $\left\{X_{t}\right\}_{t \in T} \subset \mathcal{T}_{\mu}^{*}$, we have $\bigcup_{t \in T} X_{t} \in \mathcal{T}_{\mu}^{*}$. It suffices to prove

\footnotetext{
Key Words: Extension of Lebesgue measure, density point

Mathematical Reviews subject classification: Primary: 28A12 Secondary: 54A09

Received by the editors January 9, 1996
} 
that $\bigcup_{t \in T} X_{t}$ is $\mu$-measurable because the inclusion $\bigcup_{t \in T} X_{t} \subset \Phi_{\mu}\left(\bigcup_{t \in T} X_{t}\right)$, when $\bigcup_{t \in T} X_{t} \in \mathcal{S}_{\mu}$, is obvious. Let $X=\bigcup_{t \in T} X_{t}$ and suppose that $X$ is bounded. Let $K$ be a segment such that $X \subset K$. We show that, for each $\varepsilon>0$, there exists a $\mu$-measurable set $C$ such that $X \subset C$ and $\mu^{*}(C \backslash X)<\varepsilon$. It suffices to snow that $X \in \mathcal{S}_{\mu}$. Fix $0<\varepsilon<l(K)$. Putting

$$
\mathcal{K}=\left\{\Delta \subset K: \mu_{*}(\Delta \cap X)>\left(1-\frac{\varepsilon}{l(K)}\right) \mu(\Delta)\right\},
$$

where $\Delta$ denotes an interval, we can easily check that the family $\mathcal{K}$ forms a Vitali covering of the set $X$. Hence we have a sequence $\left\{\Delta_{n}\right\}_{n \in \mathbb{N}} \subset K$ of pairwise disjoint intervals such that $l\left(X \backslash \bigcup_{n=1}^{\infty} \Delta_{n}\right)=0$. We show that $\mu^{*}\left(\bigcup_{n=1}^{\infty} \Delta_{n} \backslash X\right)<\varepsilon$. For every positive integer $n$, there exists a $\mu$-measurable set $B_{n}$ such that $B_{n} \subset \Delta_{n} \cap X$ and $\mu\left(B_{n}\right)>\left(1-\frac{\varepsilon}{l(K)}\right) \mu\left(\Delta_{n}\right)$. Hence

$$
\begin{aligned}
\sum_{n=1}^{\infty} \mu^{*}\left(\Delta_{n} \backslash X\right) & \leq \sum_{n=1}^{\infty} \mu\left(\Delta_{n} \backslash B_{n}\right)=\sum_{n=1}^{\infty}\left(\mu\left(\Delta_{n}\right)-\mu\left(B_{n}\right)\right) \\
& <\frac{\varepsilon}{l(K)} \sum_{n=1}^{\infty} \mu\left(\Delta_{n}\right) \leq \varepsilon .
\end{aligned}
$$

Putting $C=\left(X \backslash \bigcup_{n=1}^{\infty} \Delta_{n}\right) \cup\left(\bigcup_{n=1}^{\infty} \Delta_{n}\right)$, we have that $C$ has the desired property. Clearly the set $C$ is Lebesgue measurable.

Lemma 1 Every $\mathcal{T}_{\mu}^{*}$-open set $X$ has the form $Y \backslash Z$ where $Y \in \mathcal{L}$ and $Z \in \mathcal{I}_{\mu}$.

Proof. Let $X \in \mathcal{T}_{\mu}^{*}$. Then $X \in \mathcal{S}_{\mu}$ and $X \subset \Phi_{\mu}(X)$. According to the proof of Theorem 1, for any $\varepsilon>0$ there exists a Lebesgue measurable set $C \supset X$ such that $\mu(C \backslash X)<\varepsilon$. As a simple consequence we obtain that $X=Y \backslash Z$ where $Y \in \mathcal{L}$ and $Z \in \mathcal{I}_{\mu}$.

Lemma 2 If $\mu$ is a complete extension of Lebesgue measure, such that, for each set $X \in \mathcal{S}_{\mu}, \mu\left(X \triangle \Phi_{\mu}(X)\right)=0$, then

$$
\left\{Y \in \mathcal{S}_{\mu}: Y \subset \Phi_{\mu}(Y)\right\}=\left\{Y \subset \mathbb{R}: Y=\Phi_{\mu}(Z) \backslash U ; Z \in \mathcal{S}_{\mu}, U \in \mathcal{I}_{\mu}\right\} .
$$

Proof. If $Y \in \mathcal{S}_{\mu}$ and $Y \subset \Phi_{\mu}(Y)$, then $Y=\Phi_{\mu}(Y) \backslash\left(\Phi_{\mu}(Y) \backslash Y\right)$ has the desired form. If $Y=\Phi_{\mu}(Z) \backslash U$ where $Z \in \mathcal{S}_{\mu}$ and $\mu(U)=0$, then we see that $\Phi_{\mu}(Z) \in \mathcal{S}_{\mu}$ and $\Phi_{\mu}\left(\Phi_{\mu}(Z) \backslash U\right)=\Phi_{\mu}\left(\Phi_{\mu}(Z)\right)=\Phi_{\mu}(Z) \supset \Phi_{\mu}(Z) \backslash U$.

We call the topology $\mathcal{T}_{\mu}^{*}$ a $\mu$-density topology. In case $\mu=l$ the topology $\mathcal{T}_{\mu}^{*}$ is the $\mathcal{T}_{d}$-topology called the density topology (cf. [4]). 
Lemma 3 If $\mu$ is a complete extension of Lebesgue measure, such that $\mathcal{S}_{\mu}=$ $\mathcal{L} \triangle \mathcal{I}_{\mu}=\left\{X \triangle Y: X \in \mathcal{L}, Y \in \mathcal{I}_{\mu}\right\}$, then

$$
\mathcal{T}_{\mu}^{*}=\left\{Y \subset \mathbb{R}: Y=\Phi_{l}(Z) \backslash U, Z \in \mathcal{L}, U \in \mathcal{I}_{\mu}\right\} .
$$

Proof. We see that, for each set $X \in \mathcal{S}_{\mu}, \mu\left(X \triangle \Phi_{\mu}(X)\right)=0$. Thus, applying Lemma 2 and taking account of the fact that, for every $\mu$-measurable set $A$ there exists a Lebesgue measurable set $B$ such that $\Phi_{\mu}(A)=\Phi_{l}(B)$, we obtain the desired equality.

Lemma 4 If $\mu$ is any complete extension of Lebesgue measure, then there exists a complete extension of Lebesgue measure $\mu^{\prime}$, such that

(1) $\mathcal{S}_{\mu^{\prime}}=\mathcal{L} \triangle \mathcal{I}_{\mu}$,

(2) $\mathcal{I}_{\mu^{\prime}}=\mathcal{I}_{\mu}$,

(3) $\mathcal{T}_{\mu^{\prime}}^{*}=\mathcal{T}_{\mu}^{*}$.

Proof. If $\mu$ is any extension of Lebesgue measure, then, for each $A \in \mathcal{I}_{\mu}$, we have that $l_{*}(A)=0$. Thus, applying the Marczewski method of the extension of Lebesgue measure (cf. [3]), we can consider the $\sigma$-field

$$
\mathcal{S}_{\mu^{\prime}}=\left\{X \triangle Y: X \in \mathcal{L}, Y \in \mathcal{I}_{\mu}\right\} .
$$

Putting $\mu^{\prime}(X \triangle Y)=l(X)$ where $X \in \mathcal{L}$ and $Y \in \mathcal{I}_{\mu}$, we correctly define a measure which is an extension of Lebesgue measure. We have thus proved condition (1). In fact, the measure $\mu^{\prime}$ is the restriction of the measure $\mu$ to $\mathcal{S}_{\mu^{\prime}}$. Now, we prove that $\mathcal{I}_{\mu}=\mathcal{I}_{\mu^{\prime}}$. Let $X$ be such that $\mu(X)=0$. Then $X=\emptyset \triangle X \in \mathcal{S}_{\mu^{\prime}}$ and $\mu^{\prime}(X)=0$. Let $X$ be such that $\mu^{\prime}(X)=0$. Then $X \in \mathcal{S}_{\mu^{\prime}}$. Hence $X=Y \triangle Z$ where $Y \in \mathcal{L}$ and $\mu(Z)=0$. We have that $0=\mu^{\prime}(X)=l(Y)$. This implies that $\mu(Y)=0$ and, finally, $\mu(X)=0$. The demonstration of the fact that $\mathcal{T}_{\mu}^{*}=\mathcal{T}_{\mu^{\prime}}^{*}$ will complete the proof.

We show that $\mathcal{T}_{\mu}^{*} \subset \mathcal{T}_{\mu^{\prime}}^{*}$. Let $X \in \mathcal{T}_{\mu}^{*}$. Then, by Lemma $1, X=Y \backslash Z$ where $Y \in \mathcal{L}$ and $Z \in \mathcal{I}_{\mu^{\prime}}$. By condition $2, Z \in \mathcal{I}_{\mu}$. This implies that $X \in \mathcal{S}_{\mu^{\prime}}$ and $\Phi_{\mu^{\prime}}(X)=\Phi_{\mu^{\prime}}(Y \backslash Z)=\Phi_{\mu^{\prime}}(Y)=\Phi_{l}(Y)=\Phi_{\mu}(Y)=\Phi_{\mu}(Y \backslash Z)=\Phi_{\mu}(X) \supset X$. Hence $X \in \mathcal{T}_{\mu^{\prime}}^{*}$. Let $X \in \mathcal{T}_{\mu^{\prime}}^{*}$. Then $X \in \mathcal{S}_{\mu^{\prime}}$ and $X \subset \Phi_{\mu^{\prime}}(X)$. At the same time $X \in \mathcal{S}_{\mu}$ and $\Phi_{\mu^{\prime}}(X)=\Phi_{\mu}(X)$. Thus $X \in \mathcal{T}_{\mu}^{*}$.

Combining Lemmas 3 and 4 , we have the following.

Theorem 2 Fo any complete extension $\mu$ of Lebesgue measure, the $\mu$-density topology $\mathcal{T}_{\mu}^{*}$ is the Hashimoto topology of the form

$$
\mathcal{T}_{\mu}^{*}=\left\{X \subset \mathbb{R}: X=Y \backslash Z, Y \in \mathcal{T}_{d}, \mu(Z)=0\right\} .
$$


Corollary 1 If $\mu_{1}$ and $\mu_{2}$ are complete extensions of Lebesgue measure and the families of $\mu_{1}$-null sets and $\mu_{2}$-null sets are identical, then $\mathcal{T}_{\mu_{1}}^{*}=\mathcal{T}_{\mu_{2}}^{*}$.

Lemma 5 For any complete extension $\mu$ of Lebesgue measure, the family $K\left(\mathcal{T}_{\mu}^{*}\right)$ of all $\mathcal{T}_{\mu}^{*}$-meager sets is identical with the $\sigma$-ideal $\mathcal{I}_{\mu}$.

Proof. Let $X \in \mathcal{I}_{\mu}$. We prove that $X$ is $\mathcal{T}_{\mu}^{*}$-nowhere dense. Let $U$ be a nonempty $\mathcal{T}_{\mu}^{*}$-open set. Then, by Theorem $2, U=Y \backslash Z$ where $Y \in T_{d}$ and $Z \in \mathcal{I}_{\mu}$. Putting $U^{\prime}=Y \backslash(Z \cup X)$, we have that $U^{\prime}$ is a nonempty $\mathcal{T}_{\mu}^{*}$-open subset of $U$ disjoint from $X$. This means that $X$ is a member of the family $K\left(\mathcal{T}_{\mu}^{*}\right)$. Now, let $X \in K\left(\mathcal{T}_{\mu}^{*}\right)$. It suffices to consider the fact that $X$ is a $\mathcal{T}_{\mu}^{*}$-nowhere dense set. Thus the $\mathcal{T}_{\mu}^{*}$-closure $\bar{X}$ is also a $\mathcal{T}_{\mu}^{*}$-nowhere dense set. We see that the set $\mathbb{R} \backslash \bar{X}=Y \backslash Z$ where $Y \in \mathcal{T}_{d}$ and $Z \in \mathcal{I}_{\mu}$. Hence $\bar{X}=(\mathbb{R} \backslash Y) \cup Z$ and $\mathbb{R} \backslash Y$ is a $\mathcal{T}_{d}$-closed set such that $\operatorname{Int}(\mathbb{R} \backslash Y)=\emptyset$ with respect to the $\mathcal{T}_{d}$-topology. This means that $\mathbb{R} \backslash Y$ is $\mathcal{T}_{d}$-nowhere dense and, thus, it is a Lebesgue null set (cf. ([5]). Finally, we conclude that $X \in \mathcal{I}_{\mu}$.

Lemma 6 For any complete extension $\mu$ of Lebesgue measure, each set having the Baire property with respect to $\mathcal{T}_{\mu}^{*}$ is the sum of a $\mathcal{T}_{\mu}^{*}$-open set and a $\mathcal{T}_{\mu}^{*}$ nowhere dense and closed set. Moreover, the family $\mathcal{B}\left(\mathcal{T}_{\mu}^{*}\right)$ of all sets having the Baire property with respect to $\mathcal{T}_{\mu}^{*}$ is identical with the family $\mathcal{L} \triangle \mathcal{I}_{\mu}$.

Proof. Let $X \in \mathcal{B}\left(\mathcal{T}_{\mu}^{*}\right)$. Thus $X=(U \backslash Y) \cup Z$ where $U \in \mathcal{T}_{\mu}^{*}$ and $Y, Z \in$ $\mathcal{K}\left(\mathcal{T}_{\mu}^{*}\right)$. By Lemma 5 , the sets $Y, Z \in \mathcal{I}_{\mu}$ and, at the same time, they are closed and nowhere dense in the topology $\mathcal{T}_{\mu}^{*}$. Hence $U \backslash Y$ is $\mathcal{T}_{\mu}{ }^{*}$-open and the set $X$ has the desired representation.

We prove that $\mathcal{B}\left(\mathcal{T}_{\mu}^{*}\right)=\mathcal{L} \triangle \mathcal{I}_{\mu}$. Let $X \in \mathcal{B}\left(\mathcal{T}_{\mu}^{*}\right)$. Thus $X=U \backslash Y$ where $U \in \mathcal{T}_{\mu}^{*}$ and $Y \in \mathcal{I}_{\mu}$. By Theorem $2, U=W \backslash Z$ where $W \in \mathcal{T}_{d}$ and $Z \in \mathcal{I}_{\mu}$. Hence $X \in \mathcal{L} \triangle \mathcal{I}_{\mu}$.

Now, we show that $\mathcal{L} \triangle \mathcal{I}_{\mu} \subset \mathcal{B}\left(\mathcal{T}_{\mu}^{*}\right)$. By Lemma $5, \mathcal{I}_{\mu} \subset \mathcal{B}\left(\mathcal{T}_{\mu}^{*}\right)$. We prove that $\mathcal{L} \subset \mathcal{B}\left(\mathcal{T}_{\mu}^{*}\right)$. Let $X \in \mathcal{L}$. Thus $X=\left(\Phi_{l}(X) \backslash\left(\Phi_{l}(X) \triangle X\right)\right) \cup$ $\left(\left(\Phi_{l}(X) \triangle X\right) \backslash \Phi(X)\right)$. We conclude that the set $X$ is the sum of a $\mathcal{T}_{\mu}^{*}$ open set and a $\mu$-null set. This implies that $X \in \mathcal{B}\left(\mathcal{T}_{\mu}^{*}\right)$. Finally, we have $\mathcal{L} \triangle \mathcal{I}_{\mu} \subset \mathcal{B}\left(\mathcal{T}_{\mu}^{*}\right)$.

Corollary $2 \mathcal{B}\left(\mathcal{T}_{\mu}^{*}\right)=\mathcal{B}\left(\mathcal{T}_{d}\right) \triangle \mathcal{I}_{\mu}$.

Corollary $3 \mathcal{B}\left(\mathcal{T}_{\mu}^{*}\right)=\operatorname{Borel}\left(\mathcal{T}_{\mu}^{*}\right)$.

Corollary $4 \operatorname{Borel}\left(\mathcal{T}_{\mu}^{*}\right)=\operatorname{Borel}\left(\mathcal{T}_{\mu}^{*}\right) \triangle \mathcal{I}_{\mu}$.

Based on Lemmas 5 and 6, we have the following assertion. 
Theorem 3 For any complete extension $\mu$ of Lebesgue measure, the topology $\mathcal{T}_{\mu}^{*}$ is the von Neumann topology associated with the measure $\mu$ if and only if $\mathcal{S}_{\mu}=\mathcal{L} \triangle \mathcal{I}_{\mu}$.

Lemma 7 If $\mu$ is any complete extension of Lebesgue measure such that for every $X \in \mathcal{S}_{\mu}, X \backslash \Phi_{\mu}(X) \in \mathcal{I}_{\mu}$, then the topology $\mathcal{T}_{\mu}^{*}$ is the von Neumann topology associated with $\mu$.

Proof. By Lemma 5, we see that the family $\mathcal{K}\left(\mathcal{T}_{\mu}^{*}\right)$ of meager sets with respect to the topology $\mathcal{T}_{\mu}^{*}$ is identical with $\mu$. Thus we need only prove that the family $\mathcal{B}\left(\mathcal{T}_{\mu}^{*}\right)$ of Baire sets is identical with $\mathcal{S}_{\mu}$. By Lemma $6, \mathcal{B}\left(\mathcal{T}_{\mu}^{*}\right) \subset \mathcal{S}_{\mu}$. Let $X \in \mathcal{S}_{\mu}$. By assumption, we have that $X \backslash \Phi_{\mu}(X) \in \mathcal{I}_{\mu}$ and moreover, it is easy to see that $\Phi_{\mu}(X) \backslash X \in \mathcal{I}_{\mu}$. Hence $\Phi_{\mu}(X)$ is the $\mu$-measurable set. Putting $X=\left(X \cap \Phi_{\mu}(X)\right) \cup\left(X \backslash \Phi_{\mu}(X)\right)$, we have that $X \backslash \Phi_{\mu}(X) \in \mathcal{K}\left(\mathcal{T}_{\mu}^{*}\right)$ and $X \cap \Phi_{\mu}(X) \subset \Phi_{\mu}(X)=\Phi_{\mu}\left(X \cap \Phi_{\mu}(X)\right)$. The last assertion means that $X \cap \Phi_{\mu}(X) \in \mathcal{T}_{\mu}^{*}$ and we have obtained that the set $X$ has the Baire property with respect to the topology $\mathcal{T}_{\mu}^{*}$.

Theorem 4 If $\mu$ is any complete extension of Lebesgue measure, then the condition that for every $X \in \mathcal{S}_{\mu}, X \triangle \Phi_{\mu}(X) \in \mathcal{I}_{\mu}$, holds if and only if $\mathcal{S}_{\mu}=\mathcal{L} \triangle \mathcal{I}_{\mu}$.

Proof. The necessity is the consequence of Theorem 3 and Lemma 7.

The sufficiency is the consequence of the Lebesgue density theorem.

Now, we turn our attention to the family of continuous functions with respect to $\mu$-density topology.

Applying Theorem 4 in [4], we conclude the following property.

Proposition 1 If $(X, \mathcal{T})$ is a topological space and $\mathcal{I}$ is an arbitrary $\sigma$-ideal of subsets of $X$ free from nonempty $\mathcal{T}$-open sets and such that the family of sets $\mathcal{T}-\mathcal{I}=\{Y \subset X: Y=W \backslash Z, W \in \mathcal{T}, Z \in \mathcal{I}\}$ forms a topology (called the Hashimoto topology), then the family of all real continuous functions in the topology $\mathcal{T}$ is identical with the family of all real continuous functions in the topology $\mathcal{T}-\mathcal{I}$.

As an easy application of this proposition and Theorem 2 we have the following.

Theorem 5 For any complete extension $\mu$ of Lebesgue measure, the family of all real functions which are continuous in the $\mu$-density topology is identical with the family of all approximately continuous functions. 


\section{References}

[1] H. Hashimoto, On the *topology and its application, Fund. Math., 91 (1976), 5-10.

[2] A .B. Kharazishvili, The invariant extensions of the Lebesgue measure, Tbilisi 1983. (in Russian)

[3] E. Marczewski, Sur l'extension de la mesure lebesguienne, Fund. Math., 25 (1935), 551-558.

[4] N. F. G. Martin, Generalized condensation points, Duke Math. J., 28 (1961), 507-514.

[5] J. C. Oxtoby, Measure and Category, Springer-Verlag, 1971. 